\title{
Effect of juvenile pretraining on adolescent structural hippocampal attributes as a substrate for enhanced spatial performance
}

\author{
Robin J. Keeley, Brianne C. Wartman, Alexander N. Häusler, and Matthew R. Holahan ${ }^{1}$ \\ Department of Psychology, Institute for Neuroscience, Carleton University, Ottawa, Ontario K1S 5B6, Canada
}

\begin{abstract}
Research has demonstrated that Long-Evans rats (LER) display superior mnemonic function over Wistar rats (WR). These differences are correlated with endogenous and input-dependent properties of the hippocampus. The present work sought to determine if juvenile pretraining might enhance hippocampal structural markers and if this would be associated with spatial processing improvements. Male and female WR and LER were either handled or trained on a water maze task from postnatal day 16 (p16) to p26 (pretraining). All animals were then trained on the task from p40 to p44 followed by immunohistochemical assessment of synaptophysin (to mark presynaptic terminals), MAP-2 (to mark dendrites), and the phosphorylated (activated) form of the extracellular signal-regulated kinase-1 (pERK1) in the hippocampus. From p19 to p20, LER (both male and female) showed a dramatic improvement in locating the hidden platform compared to their WR counterparts. On the first day of training at p40, all pretrained groups showed shorter latencies to locate the platform compared to groups without pretraining. Over the next $4 \mathrm{~d}$, only pretrained male LER showed enhanced memory. Immunohistochemical analysis revealed fewer pERK1-labeled neurons in the CA3 hippocampal region in all pretrained groups and fewer pERK1-labeled neurons in the CAl region of pretrained male LER. Pretrained male LER also showed more MAP-2 staining in CAl and dentate gyrus regions. Synaptophysin staining revealed a pattern of axonal redistribution in the $\mathrm{CA} 3$ region in the pretrained groups. Results suggest a pattern of structural hippocampal alterations that may help to identify network malleability following pretraining protocols.
\end{abstract}

[Supplemental material is available online at http://www.learnmem.org.]

The complexity of spatial learning and memory in animals is shown by the involvement of a distributed network of brain regions and underlying processes that, when interfered with, disrupt spatial memory function. Lesions or temporary inactivations of the dorsal hippocampus reliably disrupt performance on spatial tasks (Morris et al. 1982; Sutherland et al. 1982, 1983; McDonald and White 1994; Moser et al. 1995; Duva et al. 1997; Cassel et al. 1998; Oswald et al. 2003), suggesting a hippocampal contribution to spatial memory function. As well, impeding $N$-methyl-Daspartic acid receptor (NMDAr) function can mimic the effects of hippocampal lesions on a number of spatial tasks (Morris 1989; Robinson et al. 1989; Heale and Harley 1990; Shapiro and Caramanos 1990; Ohno et al. 1992; Bannerman et al. 1995; Riekkinen and Riekkinen 1997; Ahlander et al. 1999; Steele and Morris 1999; Holahan et al. 2005).

Under certain circumstances, animals with hippocampal lesions or NMDAr blockade can show recovered spatial learning (Saucier and Cain 1995; Whishaw et al. 1995; Cain et al. 1996; Whishaw and Jarrard 1996). One method to overcome deficits on spatial tasks is to employ pretraining protocols. For example, animals that were pretrained in the water maze and given AP5 during acquisition showed fewer sensorimotor disturbances (e.g., falling off the platform or failing to mount it) and subsequently, acquisition of the task by these animals was superior to nonpretrained animals (Morris 1989). This recovered function has been ascribed to overcoming sensorimotor deficits produced by

\footnotetext{
${ }^{1}$ Corresponding author.
}

E-mail matthew_holahan@carleton.ca; fax (613) 520-4052.

Article is online at http://www.learnmem.org/cgi/doi/10.1101/lm.1849910.
NMDAr blockade (Keith and Rudy 1990; Cain et al. 1996, 1997; Saucier et al. 1996). The possibility also exists that pretraining protocols might enhance the processing capacities of intrahippocampal networks necessary for the cognitive aspects of the task (Otnaess et al. 1999; Uekita et al. 2006; Uekita and Okaichi 2009) or extra-hippocampal networks that also contribute to spatial information processing (McDonald and White 1994, 1995; Devan and White 1999; McDonald et al. 2004; Holahan et al. 2005; Knierim 2006). In this way, pretraining would affect a mnemonic function rather than sensorimotor processes. Alternatively, improved water maze performance from pretraining procedures may be due to lowered levels of stress elicited by the water maze task. Increased levels of stress hormones, specifically cortisol, are negatively correlated with water maze performance (Roskoden et al. 2005), and manipulations, which lower the overall stress response, have been correlated with improved water maze performance (Meaney and Aitken 1985; Issa et al. 1990; Zaharia et al. 1996). For animals with no pretraining, poor performance on the water maze task could reflect elevated stress levels.

In the present study, water maze training during the juvenile period (postnatal day 16-26; p16-p26) was used as a spatial pretraining protocol. The work of Rauch and Raskin (1984) has demonstrated the beneficial effects of juvenile training in preweanling rats. In this report, training on a spatial navigation task from p16 to $\mathrm{p} 20$ resulted in water maze performance that was significantly better than chance as well as significantly better than naïve animals. Presently, a juvenile spatial pretraining protocol was used in two rat strains that have been extensively employed in brainbehavior studies: Long-Evans (LER) and Wistar (WR) rats. In direct comparisons of LER and WR, LER consistently show 
superior memory function over WR on a variety of behavioral tasks (Ambrogi Lorenzini et al. 1987; Andrews et al. 1995) occurring independent from sensorimotor differences (Holahan et al. 2006). This deficient memory function in WR has a neural correlate in the distribution of hippocampal mossy fibers (MF) to the CA3 region (Holahan et al. 2006).

The developmental time span (p16-p26) was chosen based on previous work, which showed developmental differences in LER and WR in terms of CA3 hippocampal MF distribution (Holahan et al. 2007). In both LER and WR, MF innervation of the stratum lucidum (SL) and the stratum oriens (SO) was observed at p18 and p21. By p24, the SO projection in WR was no longer detectable, whereas in LER, the SO projection seen on p21 remained and persisted to adulthood (Holahan et al. 2006). If this represents a sensitive period for hippocampal development and modification, it would be predicted that input-dependent processes during this time span would benefit hippocampal development and subsequent spatial information processing.

The current study was run on male and female LER and WR to determine the effect of a juvenile spatial pretraining protocol on later spatial processing and hippocampal structure. In this way, measures of learning could be taken during juvenile and adolescent phases with or without pretraining. After adolescent training, immunohistochemical assessment of synaptophysin, to mark presynaptic terminals, a microtubule-associated protein, MAP-2, to mark dendrites, and the phosphorylated (activated) form of the extracellular signal-regulated kinase-1 (pERK1) was undertaken in the hippocampus. The main objective of this study was to determine how pretraining during the juvenile period influenced later performance on the task and hippocampal structural features. The LER and WR strains were used as a way to fully investigate the relationship between early experience and later performance and structural change. It was hypothesized that animals benefiting most from juvenile pretraining, evidenced by enhanced spatial learning as adolescents, would also show augmented hippocampal structural elements.

\section{Results}

\section{Juvenile water maze training}

Juvenile rats (p16-p26) were trained on a modified spatial water maze task (see Fig. 1 for illustration). On Day 1, rats were given eight trials to find a hidden platform at a fixed location. For the first trial on the second day, the platform was in the same location as the previous day. The platform was then relocated for the remaining seven trials. On Day 3, a similar procedure was followed such that the platform was located in the same position for Trial 1 as it was for Trials $2-8$ on day 2 , then moved to a new location for Trials 2-8 (see Fig. 1 for illustration). For juvenile pretraining, the pattern of platform switching continued for 6 more days until p26.

Using the water maze task as illustrated in Figure 1, optimal learning should be demonstrated by lower latencies on the first trial to locate the platform as training progresses. When the platform is moved on Trial 2, animals should show longer latencies as they search for the new platform location. The latencies on Trial 3 should then be shorter than those on Trial 2. Examination of the Trials 1-3 latency data from the juvenile pretraining phase for both LER and WR from p20 to p26 did not reveal this pattern of learning (see Supplemental Fig. 1).

Latency data for the juvenile pretraining phase were averaged over the eight trials to obtain a daily average per animal. Group (LER female, LER male, WR female, WR male) averages for each day were plotted over the 11 training days as shown in Figure 2. Latency data revealed that from p16 to p19, all groups performed

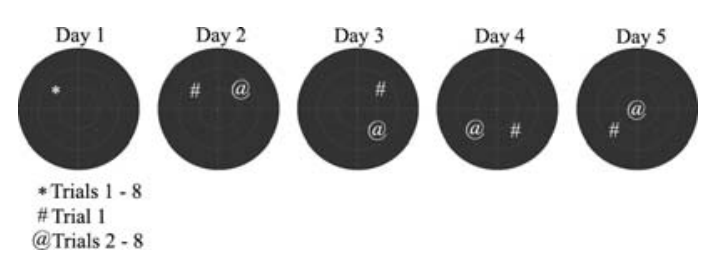

Figure 1. Platform locations across $5 \mathrm{~d}$ of testing. On the first day, the platform remained in the same position throughout all trials. On all other testing days, the platform was moved such that on the first trial, the platform was in the previous day's location. On the second through eighth trial, the platform was moved to a new and different location in the pool. (*) Represents the platform location on Day 1 Trials 1 through 8; (\#) represents platform location on the first trial on every other day; (@) represents the platform location on the second through eighth trials on every other day.

similarly on the task. From p20 to p26 (the last day of juvenile training), the LER strain (both male and female) outperformed the WR strain. A two-way repeated-measures ANOVA revealed main effects of group $\left(F_{(3,20)}=17.42, \quad P<0.001\right)$ and day $\left(F_{(10,30)}=103.26, \quad P<0.001\right)$ and a significant interaction between these factors $\left(F_{(30,200)}=3.95, P<0.001\right)$. As shown in Figure 2, p20 was the first day where obvious differences in the average daily latencies were observed. A one-way ANOVA on the p20 latency data revealed significant group differences $\left(F_{(3,20)}=\right.$ 9.47, $P<0.001$ ) with post-hoc least significant difference (LSD) tests revealing significant differences between LER and WR females $(P<0.01)$ and LER and WR males $(P<0.01)$. Withinstrain differences between genders were not detected. The differences in training latencies remained to p26 $\left(F_{(3,20)}=8.28, P<\right.$ 0.01 ), with LER females and males outperforming WR females and males, respectively $(P<0.01)$. Within-strain differences between genders were not detected on any juvenile training day (see Fig. 2).

\section{Adolescent water maze training}

\section{Female cyclicity}

Vaginal swabs were taken from adult females following vaginal opening ( $\sim$ p30) to determine phase within the estrous cycle using the lavage technique. No overall differences were observed in different phases of the estrous cycle for any behavioral measure examined. For example, average latencies on Day 1 of adolescent training for the LER females with no pretraining were $38.5 \mathrm{sec}$ (estrous), $33.8 \mathrm{sec}$ (metestrous), $46.4 \mathrm{sec}$ (diestrous), and

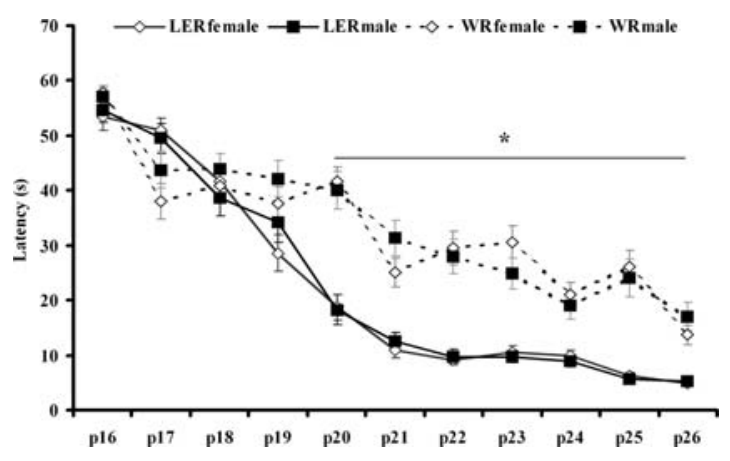

Figure 2. Latency to reach the platform averaged across eight trials of daily training for LER females, LER males, WR females, and WR males from p16 to $\mathrm{p} 26$. Significant differences were observed between LER and WR groups (regardless of gender) from p20 to p26. ${ }^{*} P<0.05$. 
$35.16 \mathrm{sec}$ (proestrous) and for the WR females with no pretraining were $38.8 \mathrm{sec}$ (estrous), $42.9 \mathrm{sec}$ (metestrous), $45.7 \mathrm{sec}$ (diestrous), and $37.3 \mathrm{sec}$ (proestrous). Because of this, estrous cycle was not considered in any analyses.

\section{Day 1}

Adolescent water maze training was carried out from p40 to p44. Groups (LER males and females; WR males and females) either had early juvenile pretraining or did not. The water maze training design is shown in Figure 1. Because the platform was located in the same position on all trials on Day 1, this day was analyzed separately from Days 2-5. Latency data for each group (LER female, LER male, WR female, WR male) were analyzed using juvenile experience as the independent factor and latency on each trial as the repeated, dependent measure (Fig. 3). All groups with juvenile pretraining showed shorter latencies overall on Day 1 than groups that did not receive juvenile pretraining (all group effects significant at 0.05; LER female, $F_{(1,8)}=31.99$, Fig. 3A; LER male, $F_{(1,9)}=7.4$, Fig. 3B; WR female, $F_{(1,9)}=10.42$, Fig. 3C; WR male, $F_{(1,9)}=34.55$, Fig. 3D). All groups except WR males (Fig. 3D) showed main effects of trial indicating that all groups (expect WR males) decreased their latencies over the eight trials on Day 1 regardless of juvenile experience (all main effects of trial significant at 0.05 except WR males; LER female, $F_{(7,7)}=4.43$; LER male, $F_{(7,7)}=6.97$; WR female, $F_{(7,7)}=2.30$; WR male, $F_{(7,7)}=1.72, P=$ 0.12). The WR females (Fig. 3C) were the only group to show an interaction between juvenile experience and trial $\left(F_{(7,63)}=3.71\right.$, $P<0.01)$. This appears to be due to the WR female group with juvenile pretraining showing rapidly decreasing latencies from Trial 1 to Trial 3, followed by longer latencies from Trials 4 to 7 (Fig. 3C).
A comparison of the percent change from the average Day 1 latencies without juvenile pretraining to the average Day 1 latencies with juvenile pretraining revealed that all groups showed similar improvements on Day 1 with juvenile pretraining (LER female: mean change $51.43 \%$, LER male: mean change $41.12 \%$, WR female: mean change $33.86 \%$, WR male: mean change $48.33 \%)$. Overall, these data recommend the conclusion that Day 1 performance was enhanced in all juvenile pretrained groups compared with those with no pretraining.

\section{Days 2-5}

Data analysis for Days 2 through 5 was carried out on Trials $1-3$ for each day, as the platform was located in one position on Trial 1 then moved to a different position for Trials 2 and 3. Latency data recorded on Trials 1 through 3 (repeated factor) for Days 2-5 (repeated factor) for each group were analyzed using juvenile experience as the between-factor (three-way ANOVA; see Fig. 4). Figure $4 \mathrm{~A}$ shows the LER female latency data. Analysis revealed only a main effect of trial $\left(F_{(2,16)}=5.79, P<0.05\right)$. Figure $4 \mathrm{~B}$ shows the latency data collected for the LER males. Three-way ANOVA on these data revealed main effects of group $\left(F_{(1,9)}=\right.$ 13.78, $P<0.01)$ and trial $\left(F_{(2,18)}=8.18, P<0.01\right)$ and, most importantly, a three-way interaction between day, trial, and group $\left(F_{(6,54)}=2.90, P<0.05\right)$. LSD post-hoc tests revealed shorter latencies in the LER males with juvenile pretraining on Trial 1 of Day $2(P<0.01)$, Trial 1 of Day $3(P<0.05)$, and Trial 2 of Day 4 $(P<0.001)$. Analyses carried out on WR females revealed no significant differences (Fig. 4C). In WR males, a day $\times$ trial interaction was the only significant finding $\left(F_{(6,54)}=2.40, P<0.05\right)$. These analyses revealed that the only group to benefit from juvenile pretraining over Trials 1-3 on Days $2-5$ was the LER males.
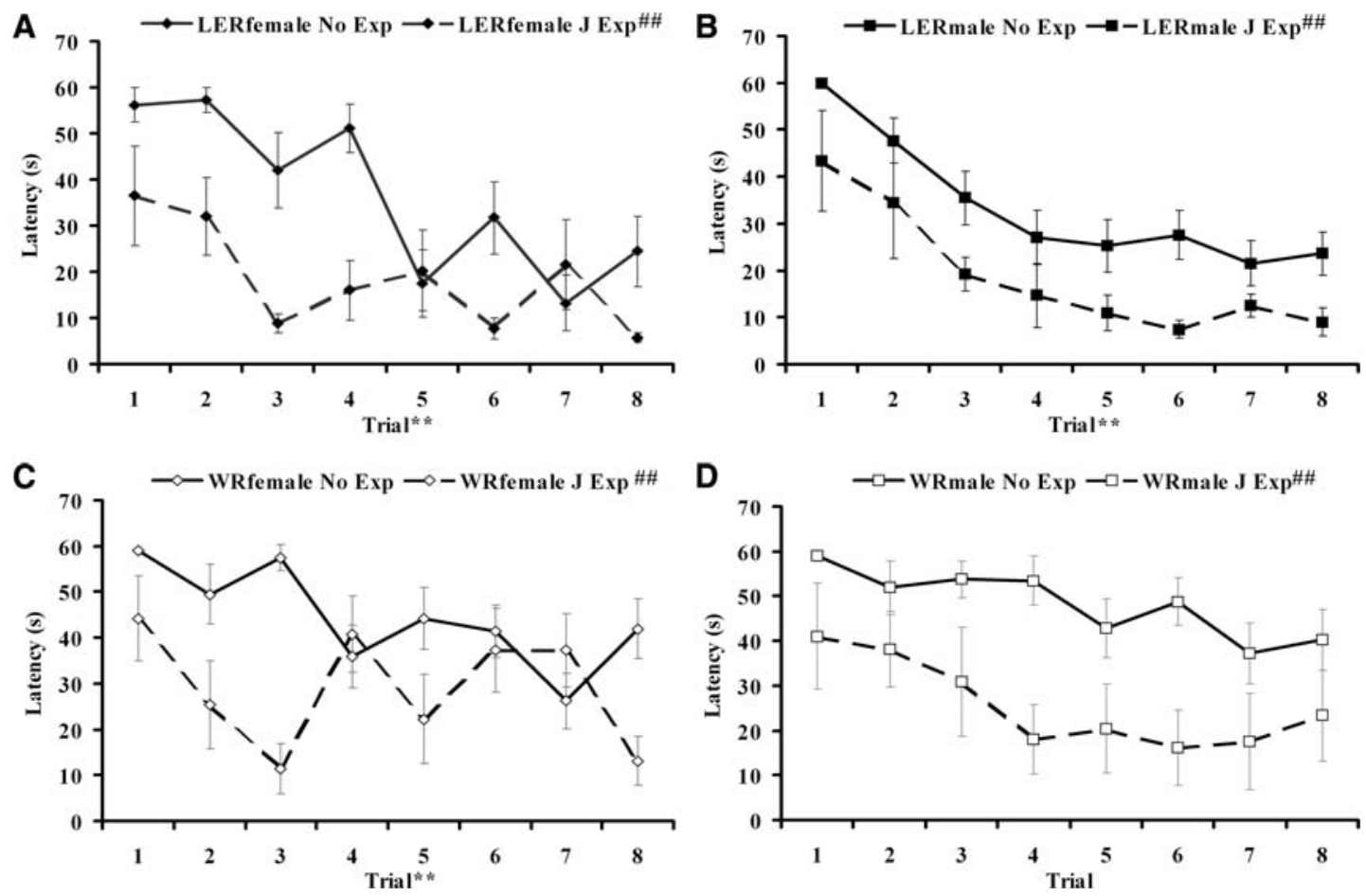

Figure 3. Latency to reach the platform on Day 1 for adolescent water maze training. (A) LER females with (J Exp) or without (No Exp) juvenile pretraining. $(B)$ LER males with or without juvenile pretraining. (C) WR females with or without juvenile pretraining. (D) WR males with or without juvenile pretraining. On Day 1, the platform was located in the same position throughout the eight trials. (\#\#) Indicates a main effect of juvenile experience $(P<0.05)$; $(* *)$ indicates a main effect of trial $(P<0.05)$. 
A comparison of the percent change from the average Day 2 Trial 1 latencies without juvenile pretraining to the average Day 2 Trial 1 latencies with juvenile pretraining revealed that LER males with juvenile pretraining showed the largest qualitative improvement (LER female: mean change $58.36 \%$, LER male: mean change $79.90 \%$, WR female: mean change $57.80 \%$, WR male: mean change $12.30 \%)$.

Because one objective of the present work was to determine if juvenile pretraining could overcome the spatial deficits as noted in WR compared with LER, latency data for the first three trials on Days 2-5 were plotted and compared for WR males with juvenile pretraining and LER males without juvenile pretraining (Supplemental Fig. 2). This comparison revealed very similar learning curves, suggesting juvenile pretraining in male WR allowed them to "catch up" to the spatial learning capacity as noted in nonpretrained LER.

It is also worthwhile to compare latency data over Trials 1-3 between juvenile animals (Supplemental Fig. 1) and adolescent animals (Fig. 4). In particular, the LER adolescents (both male and female) show the inverted latency curve with short latencies on the first trial,

longer latencies on the second trial, and short latencies on the third trial. Juvenile animals did not consistently show this pattern of behavior.

\section{Immunohistochemistry}

Forty-eight hours after the last day of adolescent water maze training, rats were euthanized, brains were removed, and the hippocampus was prepared for immunohistochemical analysis using antibodies raised against synaptophysin (to mark presynaptic terminals), MAP-2 (to mark dendrites), and the phosphorylated (activated) form of pERK1. Figure 5 shows representative images from LER and WR male animals for all stains employed.

\section{pERK1}

For pERK1 immunohistochemistry, labeled neurons were examined in the CA1, dentate gyrus (DG), and CA3 dorsal hippocampal subregions. pERK1-labeled neurons for each group (LER female, LER male, WR female, WR male) were analyzed using juvenile experience as the independent factor and hippocampal subregion as the repeated, dependent measure (Fig. 6). All groups that had juvenile pretraining showed fewer pERK1-labeled pyramidal neurons in hippocampal CA3 (Fig. 5A-D), while only the LER strain with juvenile pretraining showed fewer labeled granule cells in DG (Fig. 5A and B) and finally, only LER males with juvenile pretraining showed fewer labeled pyramidal neurons in CA1 (Fig. 5B). For the LER female group, there were main effects of group $\left(F_{(1,8)}=9.50, P<0.05\right)$ and region $\left(F_{(2,2)}=38.01, P<0.001\right)$ but no interaction. In both the DG $(P<0.05)$ and CA3 $(P<0.01)$ regions there were fewer pERK1-labeled neurons in the group that received juvenile pretraining compared with nonpretrained LER females. For the LER male group, there were main effects of group $\left(F_{(1,8)}=9.28, P<0.001\right)$ and region $\left(F_{(2,2)}=15.50, P<\right.$
$0.001)$ and a significant interaction $\left(F_{(2,16)}=8.68, P<0.001\right)$. In the CA1 $(P<0.001)$, the DG $(P<0.05)$, and CA3 $(P<0.001)$ regions there were fewer pERK1-labeled neurons in the group that received juvenile pretraining compared with nonpretrained LER males. For the WR female group, there was only a main effect of region $\left(F_{(2,2)}=37.26, P<0.001\right)$. In the CA3 $(P<0.05)$ region there were fewer pERK1-labeled neurons in the group that received juvenile pretraining compared with nonpretrained WR females. For the WR male group, there was a main effect of region $\left(F_{(2,2)}=90.08, P<0.001\right)$ and a significant interaction between region and group $\left(F_{(2,16)}=6.51, P<0.01\right)$. In the CA3 $(P<0.01)$ region, there were fewer pERK1-labeled neurons in the group that received juvenile pretraining compared with nonpretrained LER males.

\section{Synaptophysin}

Synaptophysin staining was quantitatively studied using two coronal sections from each animal. The synaptophysin-stained area in the CA3 SL and SO (Fig. 5D) was outlined and measured. The synaptophysin-stained area for each region (SO and SL) was taken as an average from both coronal levels. Comparisons between groups were made using the ratio of the stained area in SO to that in SL as in Holahan et al. (2006). The larger the ratio, the more observed synaptophysin staining in SO which is suggestive of axonal remodeling.

Multiple independent samples' $t$-tests were used to analyze synaptophysin ratios in each group (LER female, LER male, WR female, WR male) using juvenile experience as the group factor. Due to the use of four $t$-tests, the alpha level was adjusted using a Bonferoni correction $(0.05 / 4=0.01)$. LER females with or without juvenile pretraining showed similar SO:SL synaptophysin staining ratios in the CA3 region $(P=0.98$, Fig. 7A). In the remaining groups, pretraining was associated with a larger SO:SL 


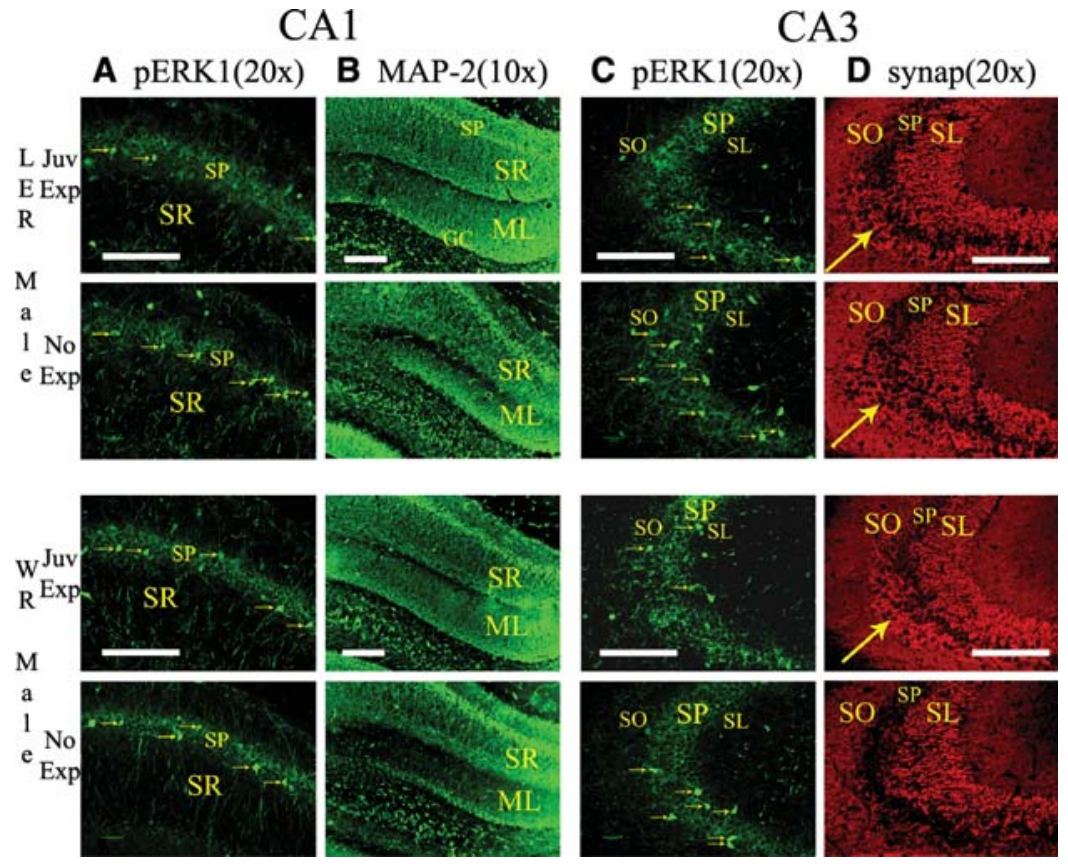

Figure 5. Representative images from LER and WR males for immunohistochemical localization of pERK1 in CA1 $(A)$, MAP-2 in CA1 and DG $(B)$, pERK1 in CA3 $(C)$, and synaptophysin in CA3 $(D)$. Only representative staining from males is shown; quantification was carried out on both males and females. Top row for each group includes sections from animals with juvenile pretraining (Juv Exp) and bottom row for each group shows sections from animals without juvenile pretraining (No Exp). pERK 1 and synaptophysin images were taken at $20 \times$ with scale bar $=200 \mu \mathrm{m}$. MAP-2 images were taken at $10 \times$ with scale bar $=200 \mu \mathrm{m}$. Arrows in $(A)$ and $(C)$ indicate pERK1-stained neurons. Arrows in $(D)$ show synaptophysin staining in stratum oriens (SO). Other abbreviations are: SR: stratum radiatum, SL: stratum lucidum, SP: stratum pyramidale, GC: granule cells, ML: molecular layer.

synaptophysin staining ratio compared with nonpretrained groups (LER males: $P<0.01$, Fig. 7B; WR females: $P<0.001$, Fig. 7C; WR males: $P<0.001$, Fig. 7D), indicating more synaptophysin staining in the CA3 SO region with juvenile pretraining.

Analysis of the raw areal measurements (millimeter squared) for synaptophysin staining in the CA3 region is shown in Supplemental Figure 3. The synaptophysin-stained area for each group (LER female, LER male, WR female, WR male) was analyzed using juvenile experience as the independent factor and hippocampal CA3 subregion (SO and SL) as the repeated measure. These analyses revealed significant interactions between group and CA3 region for all groups except the LER females (LER females: $F_{(1,8)}<1.0$, Supplemental Fig. 3A; LER males: $F_{(1,7)}=$ 20.56, $P<0.01$, Supplemental Fig. 3B; WR females: $F_{(1,8)}=$ 33.21, $P<0.001$, Supplemental Fig. 3C; WR males: $F_{(1,7)}=$ 10.07, $P<0.05$; Supplemental Fig. 3D). Compared with the nonpretrained groups, the juvenile pretrained LER male, WR female, and WR male groups showed a significantly larger synaptophysinstained area in SO (see Supplemental Fig. 3). The LER male and WR female pretrained groups also showed a significantly smaller synaptophysin-stained area in SL compared with their nonpretrained counterparts (see Supplemental Fig. 3).

\section{MAP-2}

MAP-2 is described as a ubiquitous cytoskeletal neuronal protein that maintains microtubule stability and is specifically found in dendritic arbours of neurons (Leclerc et al. 1996). The MAP-2 stain was utilized to assess structural complexity in the dendritic trees based on the premise that more MAP-2 staining would be indicative of more complex dendritic tress and enhanced neural connectivity. For MAP-2 immunohistochemistry, labeled particles were examined in the CA1 stratum radiatum (SR), the molecular layer of the DG suprapyramidal layer, and CA3 SL dorsal hippocampal subregions. MAP-2-labeled particles for each group (LER female, LER male, WR female, WR male) were analyzed using juvenile experience as the independent factor and hippocampal subregion as the repeated, dependent measure (Fig. 8). For the LER female group (Fig. 8A), there was a main effect of hippocampal region $\left(F_{(2,2)}=33.99\right.$, $P<0.001)$ and a significant interaction $\left(F_{(2,16)}=8.43, P<0.01\right)$. The LER female with juvenile pretraining showed more MAP-2 staining in the DG than LER females without juvenile pretraining $(P<0.05)$. For the LER male group (Fig. 8B), there were main effects of group $\left(F_{(1,8)}=6.65, \quad P<0.05\right)$, and region $\left(F_{(2,2)}=67.20, P<0.001\right)$, and a significant interaction $\left(F_{(2,16)}=4.77\right.$, $P<0.05)$. In the CA1 $(P<0.05)$ and the DG $(P<0.01)$ there was more MAP-2 staining in the juvenile pretrained group than the nonpretrained LER males. For the WR female group (Fig. 8C), there was only a main effect of region $\left(F_{(2,2)}=21.21, P<0.001\right)$. For the WR male group (Fig. 8D), there was a main effect of region $\left(F_{(2,2)}=119.75, \quad P<\right.$ 0.001 ) and a significant interaction between region and group $\left(F_{(2,16)}=\right.$ $3.77, P<0.05)$. No differences were detected in any hippocampal subregion between pretrained and nonpretrained WR males.

\section{Discussion}

\section{Summary}

Training on a modified spatial version of the water maze task revealed that from postnatal day 16 (p16) to p19, LER male and female and WR male and female rats took the same amount of time to reach the hidden platform. However, from p20 to p26 (the last day of juvenile training), LER (both male and female) outperformed the WR strain, demonstrating significant decreases in their latency to locate the platform. This juvenile training served as a form of spatial pretraining for task performance during adolescence (p40-p44). During adolescence, all groups that received spatial pretraining as juveniles had shorter latencies to locate the platform on Day 1 compared with nonpretrained controls. As well, all groups that received juvenile pretraining showed fewer pERK1-labeled neurons in the CA3 region of the dorsal hippocampus compared with nonpretrained controls. All pretrained groups, except LER females, also showed more synaptophysin staining in the CA3 SO compared with the nonpretrained groups. For the remaining adolescent water maze training days, the LER male group showed the most benefit from juvenile pretraining in terms of spatial retention. Pretrained LER males also showed lower levels of pERK1 staining in the CA1 region of the dorsal hippocampus and more MAP-2 dendritic staining in the CA1 SR compared with nonpretrained animals. These results suggest that juvenile pretraining may enhance the processing capacities of intrahippocampal networks necessary for the cognitive aspects of the water maze task. 

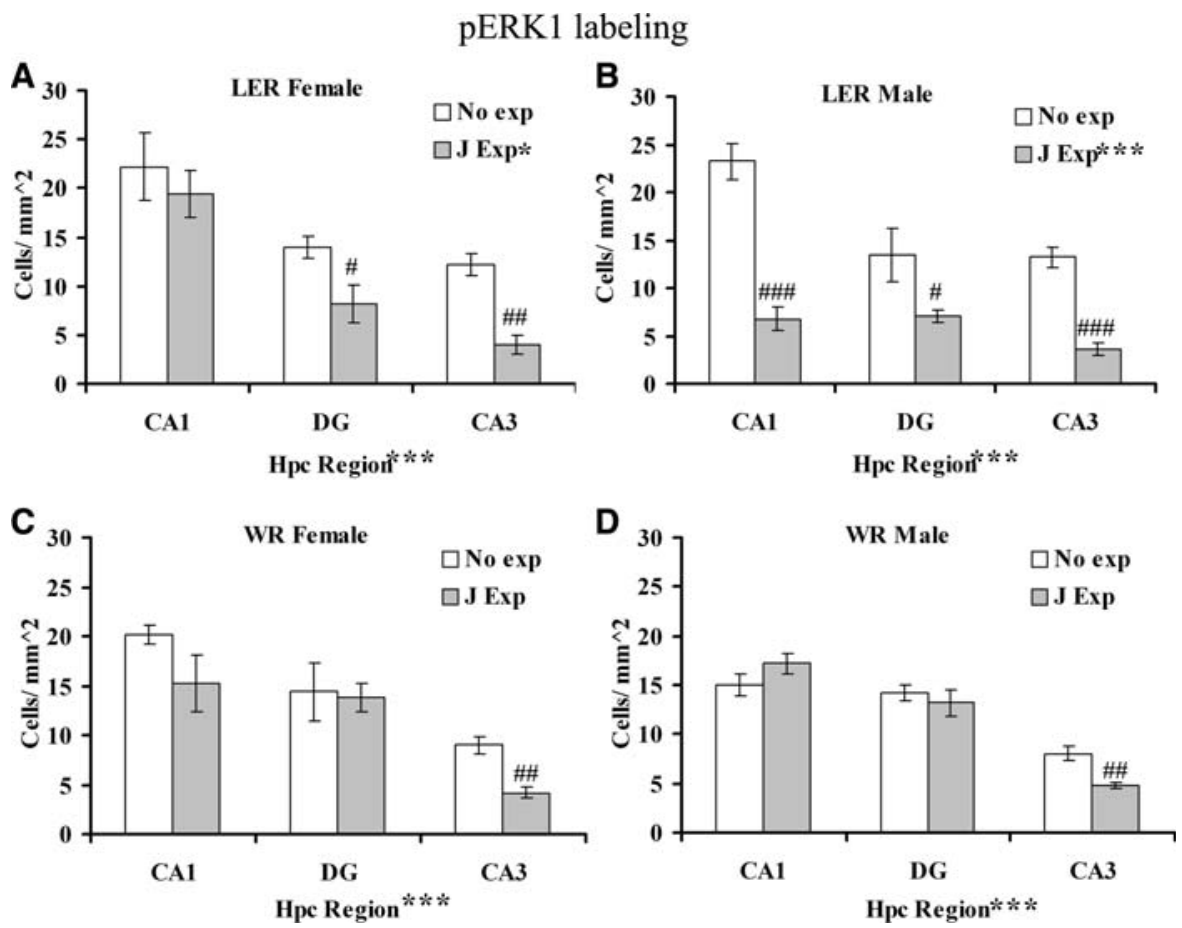

Figure 6. Quantification of pERK1 staining in the hippocampus (Hpc) CA1, dentate gyrus (DG), and CA3 for animals with (Exp) or without (No Exp) juvenile pretraining. (A) LER females. (B) LER males. (C) WR females. $(D)$ WR males. ${ }^{*} P<0.05$ (main effect of juvenile pretraining); ${ }^{* * *} P<0.01$ (main effect of region); ${ }^{\#} P<0.05,{ }^{\# \#} P<0.01,{ }^{\# \#} P<0.001$ for significant post-hoc differences within a hippocampal subregion comparing groups with or without juvenile experience.

\section{Developmental differences in spatial processing}

Juvenile training on the water maze task revealed similar performance between males and females in both LER and WR strains from p16 to p19. At p20, the strains showed a distinct split, when LER outperformed WR in terms of latency to locate the hidden platform. These results parallel earlier studies of spatial learning during development. For example, Rudy et al. (1987) showed the emergence of spatial navigation through the use of distal cues in the water maze task at p20 where, prior to this, only proximal cues were used.

One preliminary conclusion to draw from this and the current study is that a sensitive developmental period for spatial learning emerges around p20. In line with this, previous work has highlighted developmental differences in LER and WR in terms of axonal connectivity to the SO within the CA3 region of the hippocampus (Holahan et al. 2007). From p18 to p21, both strains showed a rapidly changing and expanding axonal projection throughout the entire CA3 region to SO. By p24, these changes remained in the SO of the LER, whereas in WR, at p24, there was a less-pronounced axonal connectivity pattern to SO. Thus, although LER and WR show a sensitive period for axonal projections to the SO CA3 region between p18 and p21, the WR connectivity pattern to $\mathrm{SO}$ appears to retract leaving minimal axonal innervation, a pattern that persists to adulthood (Holahan et al. 2006). In sum, the behavioral and anatomical results suggest a developmental time period, which seems to be especially important for hippocampal development and spatial processing.

\section{Gender differences}

Previous studies have demonstrated that if the estrous phase is not taken into consideration, few differences are observed between LER males and females (Warren et al. 1995; Warren and Juraska
1997). During juvenile pretraining, there were no effects of gender, suggesting that gender may not contribute to spatial processing during the juvenile period. It is interesting to note the qualitative pattern of Day 1 learning between the two genders independent of strain (Fig. 3). The latencies on Day 1 during later trials for females with and without juvenile pretraining overlap, whereas the latencies for males with juvenile pretraining are consistently lower than those for males with no pretraining. This was the only striking difference noted between the genders.

\section{Adolescent water maze performance on Day 1}

Rats were tested during the adolescent period ( $\mathrm{p} 40$ ) to determine the behavioral and anatomical effect of the juvenile pretraining. Rats were tested as adolescents rather than adults (p90) because in WR, Gaarskjaer $(1985,1986)$ reported that at $\mathrm{p} 15$, the hippocampal axonal distribution to CA3 reflects that observed in the adult. Other WR findings (Holahan et al. 2007) show that between p18 and p24, hippocampal axonal projections to CA3 go through a period of outgrowth and retraction but are complete by p24. In LER, hippocampal axonal projections to CA3 appear to be fully developed by p21. Amaral and Dent (1981) reported that at p28, the axonal projection to CA3 in LER was for the most part, fully developed, similar to Holahan et al (2007). This suggests that the hippocampus is fully developed by $\mathrm{p} 40$.

All juvenile pretrained groups showed decreased latencies on Day 1 of adolescent training compared with nonpretrained
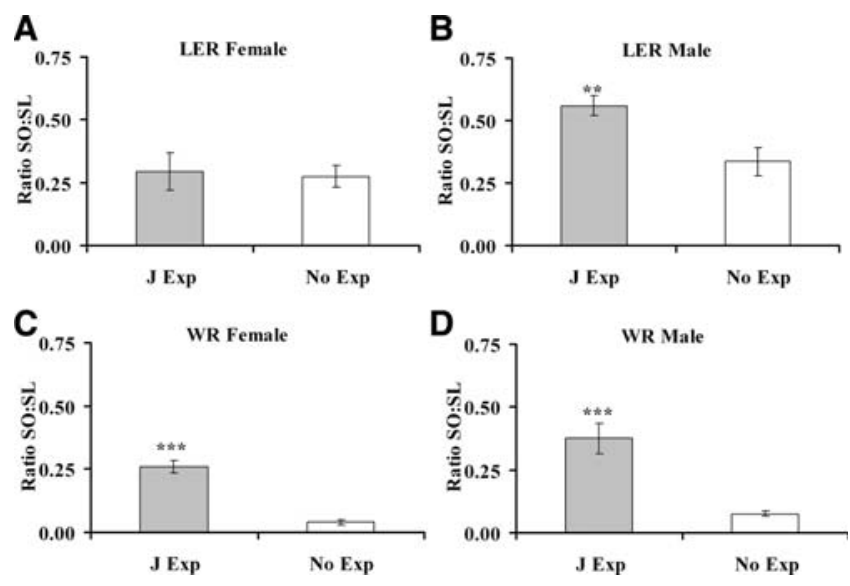

Figure 7. Quantification of synaptophysin staining in the dorsal CA3 hippocampus for animals with (Exp) or without (No Exp) juvenile pretraining. (A) LER females. (B) LER males. (C) WR females. (D) WR males. Comparisons were run within groups on the ratio of synaptophysin staining in the stratum oriens (SO) to the stratum lucidum (SL) using juvenile experience as the group factor. All groups except the LER females showed a larger SO:SL ratio with juvenile pretraining compared with groups without juvenile pretraining $\left({ }^{* *} p<0.01\right.$ and $\left.{ }^{* * *} p<0.001\right)$. 

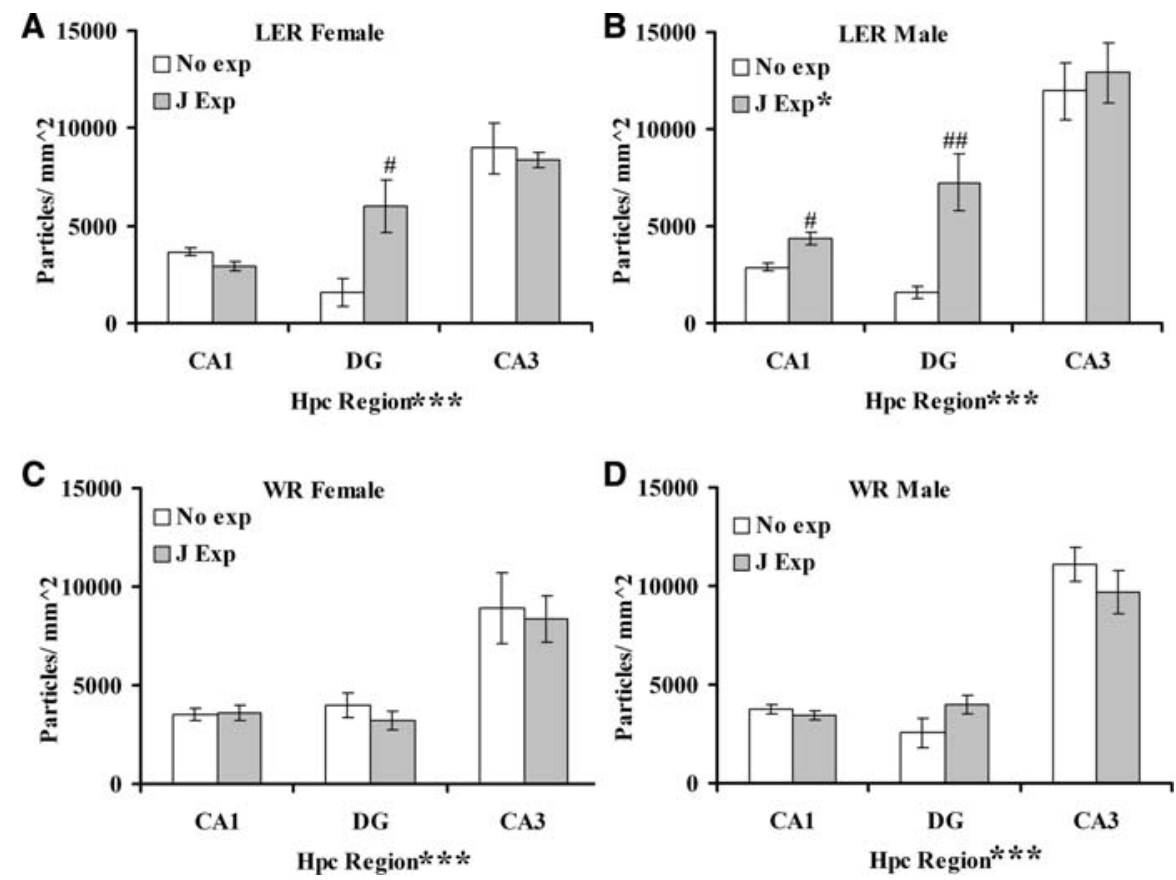

Figure 8. Quantification of MAP-2 staining in dorsal hippocampus ( $\mathrm{Hpc}$ ) CA1 SR, DG molecular layer, and CA3 SL for animals with (Exp) or without (No Exp) juvenile pretraining. (A) LER females. (B) LER males. (C) WR females. (D) WR males. ${ }^{*} P<0.05$ (main effect of juvenile pretraining); ${ }^{* * *} p<0.01$ (main effect of region); ${ }^{\#} P<0.05,{ }^{\# \#} P<0.01$ for significant post-hoc differences within a hippocampal subregion comparing groups with or without juvenile experience.

groups. Differences observed in latencies on Day 1 may result from improved swimming ability (performance factors) or task familiarity. As such, physical activity alone can enhance water maze performance (Fordyce et al. 1994). Those groups with no juvenile pretraining may have demonstrated poor performance on Day 1 as a result of increased stress elicited from this task. Chronic stress and high levels of corticosterone are negatively correlated with water maze spatial performance (Sandi et al. 2003; Roskoden et al. 2005). Juvenile pretraining may have habituated the animals to the stressful aspects of the task that occur during the first day of training in nonpretrained animals and allowed better performance independent from spatial processing ability (Meaney and Aitken 1985). Future research should examine the role of corticosterone receptors in the hippocampus following juvenile pretraining, such that blocking or stimulating these receptors would be hypothesized to alter the behavioral profile on Day 1.

\section{Adolescent water maze performance Days 2-5}

The behavioral design was hypothesized to allow for the examination of memory recall (ability of rats to recall the previous day's position), behavioral flexibility (searching for the platform in other locations once it has been moved), and acquisition of new information (the ability of rats to acquire the new platform position as tested within a day). On Day 2 (and subsequent days), optimal learning would be demonstrated with lower latencies on the first trial to locate the platform, providing an indication of reference memory recall. LER males with juvenile pretraining showed the lowest latencies on this metric. When the platform was moved on Trial 2, animals should show longer latencies as they search for the new platform location. LER males with juvenile experience showed longer latencies on Trial 2 compared with Trial 1 of Day 2 with no other groups showing this extended search pattern at this time point. The latencies on Trial 3 should then be shorter than those on Trial 2, a critical test of behavioral flexibility. The LER males with juvenile pretraining showed this inverted V-shaped pattern as early as Day 2, suggesting optimal performance was achieved and showing that LER males benefited the most from juvenile pretraining.

Examination of the trial-by-trial learning curves during juvenile pretraining using this behavioral design (Supplemental Fig. 1) showed that no juvenile groups displayed the inverted V-shaped pattern. While both strains clearly showed decreasing latencies over days and the LER strain showed latencies of under $10 \mathrm{sec}$ to locate the platform by the last $2 \mathrm{~d}$ of training, no groups showed a consistent pattern of longer latencies on Trial 3. The differences between juvenile and adolescent latency patterns may reflect a differential developmental time course of brain structures related to spatial and working memory function. As such, this task could be used to examine the development of the hippocampus and resultant behavioral output as well as examine when other neuronal structures demonstrate functional output during development. For example, it could be useful in the examination of the development of the rodent prefrontal cortex, which is known for its role in behavioral flexibility (for review, see Kolb and Robbins 2003).

One might argue that differences in performance on the last day of juvenile pretraining could account for the differences observed in the adolescent phase. However, LER males and females showed similar latencies to reach the platform on the last day of juvenile pretraining, suggesting that both groups had acquired the task similarly by the end of training. This suggests that the level of learning alone during juvenile pretraining cannot account for the enhanced spatial processing in the LER males.

\section{Immunohistochemistry}

Three stains were carried out to examine structural aspects of the dorsal hippocampus hypothesized to be affected by juvenile pretraining. The first was a stain for the phosphorylated form of the extracellular regulated kinase 1 (pERK1). Evidence suggests that phosphorylation of ERK1 is involved in cell adhesion (Adams et al. 2000), highlighting its use as a marker for cells undergoing or that have undergone some growth process. Additional work has shown that knockout of ERK1 enhances plasticity in the striatum but impairs plasticity in the hippocampal CA1 region (Mazzucchelli et al. 2002). Along with this, ERK1 seems to be involved in dendritic spine plasticity and outgrowth following activity-dependent processes (Wu et al. 2001; Goldin and Segal 2003).

All pretrained groups showed decreased pERK1 staining in dorsal hippocampal CA3 pyramidal neurons; pretrained LER males and females showed less pERK1 staining in the DG, and only pretrained LER males showed fewer pERK1-stained neurons in the CA1 region. Compared with the nonpretrained group, the pretrained LER males and females showed more dendritic staining in the DG molecular layer, and the pretrained male LER showed 
more dendritic staining in the CA1 SR. This suggests that in the pretrained animals, ERK1 may have been phosphorylated during juvenile pretraining and set into motion structural alterations that persisted into adolescence. In the nonpretrained animals, adolescent training would serve as the input-dependent process to elevate ERK1 phosphorylation that would presumably result in structural modifications at some later time point.

An antibody directed at synaptophysin was used to detect the distribution of axonal terminals in the dorsal hippocampus CA3 region. There is increasing evidence that the axonal input to CA3 may regulate spatial learning and memory by remodeling its synaptic connections. Previous work has shown that spatial water maze training is associated with a larger axonal projection to the dorsal hippocampal CA3 SO of adult WR (Ramirez-Amaya et al. 1999, 2001; Holahan et al. 2006; Rekart et al. 2007a,b) and LER (Holahan et al. 2006). This effect is specific to hidden platform spatial memory tasks; as cued, visible platform learning does not promote such growth (Rekart et al. 2007a).

All pretrained groups, except the LER females, showed more synaptophysin staining in the CA3 SO, suggesting that remodeling of axonal terminal fields took place after juvenile pretraining. While there are likely to be genetic differences between the two strains giving rise to the different patterns of axonal distribution to CA3, it is also clear that these potential genetic differences do not confer a life sentence of a diminished axonal distribution to SO. With additional stimulation provided by the juvenile pretraining method, the axonal innervation to SO in WR resembles the innervation observed in nonpretrained LER. This redistribution in WR appears to persist and have functional results by enhancing learning during the adolescent phase to the level of nonpretrained LER.

One possible explanation for the observed changes in axonal distribution to CA3 as a result of early juvenile experience would be enhanced neurogenesis in the DG (assuming that some of the axonal changes in CA3 reflect MF inputs). Based on the premise that neurogenesis in the DG leads to growth of dendrites and axons, and that these processes make functional synapses, some of which may project to CA3 (Hastings and Gould 1999; Hastings et al. 2002), it would be reasonable to suggest that juvenile pretraining enhances neurogenesis leading to additional axonal inputs to CA3 as observed in adolescence. This explanation is similar to environmental enrichment results whereby enrichment up-regulates hippocampal neurogenesis and improves water maze performance (Kempermann et al. 1997; Nilsson et al. 1999; van Praag et al. 1999, 2000; Brown et al. 2003). With enhanced neurogenesis in the pretrained juveniles, new circuits would be formed during this sensitive developmental period, leading to enhanced spatial processing later in life as previously argued by Tashiro et al. (2007). It should be noted that in pretrained groups showing changes in axonal distribution to CA3, while there is an increase in SO synaptophysin staining, there is a concomitant decrease in SL staining (Supplemental Fig. 3), resulting in similar totalstained areas between groups with and without experience. One might predict that if newly generated granule cells were the main factor influencing the changes in axonal input to CA3, there would be a greater stained area in pretrained animals. As this was not the case, it suggests the alternate explanation that remodeling of all-ready present axons may have occurred in response to juvenile pretraining.

LER males with juvenile pretraining demonstrated the most benefit in spatial recall, showing an "inverted V" pattern of behavior $1 \mathrm{~d}$ earlier than those with no pretraining. LER males with juvenile pretraining also showed a large reduction in pERK1 staining in CA1 compared with LER with no juvenile pretraining. No other group showed such a dramatic effect. Finally, LER showed the most amount of MAP-2 staining in the CA1 SR region. This pattern of results suggests that ERK1 phosphorylation as a result of juvenile pretraining sets into motion dendritic structural plasticity in the CA1 region that may serve to further refine or facilitate spatial recall.

The maintenance of synaptic plasticity at the postsynaptic element has long been associated with dendritic outgrowth. Anatomical analysis of the numbers and fine structures of spines suggested that a number of input-dependent procedures produce an increase in the size of the postsynaptic density (reviewed by Edwards 1995). Early studies (Volkmar and Greenough 1972; Greenough and Volkmar 1973; Greenough et al. 1973, 1979; Boothe et al. 1979) found that exposing animals to enriched environments was associated with an increase in the numbers of dendritic arborizations (spines) similar to what was presently observed in juvenile pretrained male LER.

Outgrowth of dendritic spines may underlie the persistent changes brought about by synaptic potentiation (Chen et al. 2004). In hippocampal slices, LTP induction in CA1 synapses leads to the appearance of new postsynaptic dendritic spines (Engert and Bonhoeffer 1999), while no spine outgrowth occurred in areas where potentiation was blocked. Visualization of hippocampal CA1 neurons expressing a green fluorescent protein showed that high-frequency synaptic stimulation enhanced postsynaptic spine growth that was both long lasting and localized to the stimulated dendritic region (Maletic-Savatic et al. 1999). Disruption of actin polymerization (Dunaevsky et al. 1999) impaired the appearance of dendritic protrusions, suggesting that actin may play an important role in dendritic outgrowth. The CA1 dendritic changes seen in the present work in pretrained male LER may further facilitate the information processing capacities of hippocampal networks, thereby enhancing spatial processing ability.

The findings reported indicate that the current spatial pretraining protocol facilitates structural changes within hippocampal networks which may provide a substrate for subsequent enhanced memory function. One such pathway, shown to be altered by juvenile pretraining in the present work, includes the CA3 synapse, a network that supports plasticity in an NMDAr-independent manner (Bortolotto et al. 2005). Findings may provide an alternate view as to why pretraining protocols overcome deficits produced by NMDAr blockade (Keith and Rudy 1990; Cain et al. 1996, 1997; Saucier et al. 1996) or LTP saturation (Otnaess et al. 1999). It may be the case that pretraining facilitates the formation of redundant or wholly separate hippocampal networks that can support learning in the face of NMDAr blockade or NMDAr-dependent LTP saturation.

\section{Materials and Methods}

\section{Subjects}

Pregnant LER $(n=3)$ and WR $(n=3)$ female rats were purchased from Charles River Laboratories (St. Constant, Quebec, Canada) and singly housed. All pups were used (11 LER females, 12 LER males, 12 WR females, and 12 WR males) for either Experiment 1 or Experiment 2. The day the pups were born was marked as p0. Pups were weaned at $21 \mathrm{~d}$ of age (p21) and group-housed. All animals were housed in a temperature controlled vivarium in polycarbonite cages with a 12-h light-dark cycle (lights on $0800 \mathrm{~h}$, lights off $2000 \mathrm{~h}$ ). Food and water were provided ad libitum. All experiments were conducted in accordance with the Canadian Council on Animal Care (CCAC) guidelines and approved by the Carleton University Animal Care Committee.

\section{Vaginal swabs}

Vaginal swabs were taken from adult females following vaginal opening $(\sim$ p30) to determine phase within the estrous cycle using 
the lavage technique. Swabs were taken at the same time of day. Slides of samples were examined using an Olympus BX-61 microscope. Estrous phase was determined as previously described (Marcondes et al. 2002).

\section{Apparatus}

Different pool and platform sizes were used for juvenile and adolescent water maze procedures. Both pools were opaque, white polypropylene. The water in the pools was made opaque with the addition of powdered, nontoxic poster paint. Water temperature averaged $21^{\circ} \mathrm{C}$ throughout behavioral testing. In both pools, the platform was submerged $2.0 \mathrm{~cm}$ below the water surface. Distal visual cues were present during all trials. Rat movements in the pool were tracked using the HVS Image 2100 Tracking System. After each training trial, animals were dried off and placed into a heated holding cage. Following the final trial on each day, every animal was dried with a towel and placed in another holding cage on a heating pad in the housing room for 10 to $15 \mathrm{~min}$ before being returned to their home cage.

\section{Behavioral procedure}

Both juvenile and adolescent animals underwent similar training procedures with the exception of the number of training days. All animals were given eight trials a day. Each trial began from a different start point on the pool's perimeter and the same sequence of start locations was used each day. On the first day, the platform remained in the same quadrant for all eight trials. For the remaining days, for Trial 1 , the platform remained in the same position as the previous days' Trials 2-8. On the second through eighth trial, the platform was moved to a different quadrant (see Fig. 1 for illustration). Latency and path length to reach the platform were recorded for all trials. If an animal did not reach the platform within $60 \mathrm{sec}$, they were guided to it. Animals remained on the platform for 30 sec before being removed and placed into a holding cage for an additional $30 \mathrm{sec}$.

\section{Juvenile training}

Pups (six each of LER males and females and six each of WR males and females) were trained in the water maze for $11 \mathrm{~d}$ (from p16 to p26) using the procedure described above.

The water maze was a white, circular, polypropylene pool measuring $124 \mathrm{~cm}$ in diameter, $31 \mathrm{~cm}$ in height, and filled with water to a depth of $25 \mathrm{~cm}$. The platform was made of clear Plexiglas and measured $11 \mathrm{~cm}$ in diameter.

\section{Adolescent training}

Five each of the LER females, LER males, WR females, and WR males that were used in Experiment 1 were retrained on the water maze task at $40 \mathrm{~d}$ of age. These animals were compared with animals with no juvenile water maze experience of the same age (five LER females, six LER males, six WR females, six WR males).

For this experiment, the water maze pool measured $155 \mathrm{~cm}$ in diameter, $60 \mathrm{~cm}$ in height, filled to a depth of $37.5 \mathrm{~cm}$. The platform was made of clear Plexiglas measuring $14 \mathrm{~cm}$ in diameter. Testing was conducted for $5 \mathrm{~d}$.

\section{Immunohistochemistry}

Rats were euthanized $2 \mathrm{~d}$ after the completion of adolescent water maze testing (p46) using $\mathrm{CO}_{2}$ followed by decapitation. Brains were removed and immersion fixed with 4\%-paraformaldehyde in $0.1 \mathrm{M}$ phosphate-buffered saline (PBS) overnight at $4^{\circ} \mathrm{C}$. This solution was replaced with $30 \%$ sucrose in $0.1 \mathrm{M}$ PBS the following day, and brains were stored at $4^{\circ} \mathrm{C}$ until sectioning. Brains were hemisected and right hemispheres were sectioned at $20 \mu \mathrm{m}$ on a Leica CM1900 cryostat. Sections from the right dorsal hippocampus were stored in a $0.1 \%$ sodium azide solution in $0.1 \mathrm{M}$ phosphate buffer $(\mathrm{PB})$ at $4^{\circ} \mathrm{C}$.

For immunohistochemistry, sections were washed for $15 \mathrm{~min}$ in a $0.2 \%$ Triton-X/0.01 M phosphate-buffered saline (T-PBS) then blocked in a $1 \times$ animal free blocker (Vector)/ T-PBS solution for $1 \mathrm{~h}$ at room temperature. Incubation in the primary antibody (mouse anti-pERK1 from Abcam, 1:5000; rabbit antisynaptophsin from Chemicon/Mililpore, 1:2500; mouse anti-MAP-2 from Chemicon/Millipore, 1:10,000) occurred overnight at room temperature. The following day, tissue was washed in T-PBS for $15 \mathrm{~min}$ followed by a 2-h incubation in the secondary antibody (1:500 goat anti-mouse 488 for pERK1; 1:500 goat anti-rabbit 594 for synaptophysin; both from Molecular Probes; 1:500 biotinylated goat anti-mouse for MAP-2). For MAP-2 staining, tissue went through an additional incubation in an avidin-biotinylated complex (ABC Elite kit; Vector Laboratories) and sections were reacted with $0.25 \% 3,3^{\prime}$-diaminobenzidine tetrahydrochloride (DAB) solution in $\mathrm{PB}$ containing $0.0025 \% \mathrm{H}_{2} \mathrm{O}_{2}$ for $6 \mathrm{~min}$. All tissue was given a final rinse in 0.01 M PBS ( $\mathrm{pH} 7.4$ ) for $15 \mathrm{~min}$ and mounted on glass slides. pERK1 and synaptophysin-stained sections were coverslipped with glass coverslips adhered with Fluormount (Sigma). MAP-2 sections were dehydrated and coverslipped with glass coverslips adhered with Permount (Sigma).

\section{Statistical analysis}

Latency and path length were found to be highly correlated, indicating little differences in these metrics. Therefore, only latency data were considered for analyses.

For juvenile water maze latency data, a two-way repeatedmeasures ANOVA was conducted with day as the repeated measure and group (LER male, LER female, WR male, WR female) as a between-subjects measure. One-way ANOVAs were run on selected days with LSD post-hoc tests to determine when specific group differences emerged.

For adolescent water maze latencies, two main analyses were carried out within each group. The first analysis examined mean latencies for Trials 1-8 (repeated factor) to reach the platform on Day 1 using juvenile experience as the between-factor for each group (LER male, LER female, WR male, WR female). This analysis was carried out because the platform remained in the same location for all eight trials. The second analysis was carried out on latency data recorded on Trials 1 through 3 for Days 2-5 for each group using juvenile experience as the between-factor (three-way ANOVA). The analysis was carried out on Trials 1-3 as the platform was located in one position on Trial 1 then moved to a different position for Trials 2 and 3. LSD post-hoc tests were run to determine specific differences on each trial using juvenile experience as the independent factor.

\section{pERKI quantification}

For each stained section, fluorescent images of the CA1 region and the DG were captured at $10 \times$ magnification while the CA3 region was captured at $20 \times$ magnification using a Retiga-2000R camera (QImaging, BC) and an Olympus BX61 research microscope (Olympus-Canada). Regions of interest were selected and traced (freehand) with reference to Paxinos and Watson (1998). When the entire CA1 region or DG could not be captured in a single image, a sample of the area was acquired. In this way, the CA1 sample region was identified by a string of pyramidal neurons that could be viewed through the camera lens when the most superior point of the hippocampus was centered horizontally and vertically. DG counts were carried out on the two blades of neurons that were viewable when the end of the superior blade reached the lateral edge of the camera window. Images were acquired using InVitro version 3.2.2 (Media Cybernetics) and pERK1/ 2 cell counts were obtained using Image-Pro Analyzer version 6.2.1.491 (Media Cybernetics).

Cells expressing pERK1 within each region of interest were identified on the basis of staining intensity. To ensure that the image intensity values were unaffected by any digital adjustments, the camera's exposure corrections remained at their default linear settings. Gain and binning were also set to their default values (1.0 and 1, respectively) and the exposure value was held constant. 
Stained cells were segmented out by manually adjusting the grayscale threshold for each section. Threshold values ranged from 1500 to 3450 (mean $=2405, \mathrm{SD}=345.60)$, depending on the intensity of the staining. The mean diameter of each segmented object was calculated by averaging the length of all diameters that could be measured at two-degree intervals around each object's perimeter. Objects with a mean diameter of $<10 \mu \mathrm{m}$ were filtered out to increase the likelihood that only pyramidal neurons or granule cells were included in the cell count. Each of the remaining segmented objects was then inspected by an experimenter who excluded objects that were obviously composed of stained background material. The final count was divided by the area of the region of interest to obtain a measure of cell density, which was recorded in number of pERK1-labeled cells per millimeter squared. Two sections from each animal were counted, and the mean of every two corresponding density values was entered into the analysis as a single datum point.

\section{Synaptophysin quantification}

For stained sections, fluorescent images of the CA3 region were captured at $20 \times$ magnification using a Retiga-2000R camera (QImaging, BC) and an Olympus BX61 research microscope (Olympus-Canada). Two coronal sections (140 $\mu \mathrm{m}$ between sections) were sampled from the dorsal hippocampus starting 200 $\mu \mathrm{m}$ from the initial blossoming of the hippocampal CA3 pyramidal cells (approximately $-1.8 \mathrm{~mm}$ from bregma). Area measurements for each region of interest were taken as averages from both coronal levels. The areas of the SL and the SO were estimated by outlining the synaptophysin-positive region. Synaptophysinpositive staining was defined as having an intensity value twice that of the background as measured on the SR of the CA3 region. Comparisons between groups were made using the ratio of the area in SO to that in SL to account for size variations between individual animals. An experimenter who was blind to group assignment carried out all analyses.

\section{MAP-2 quantification}

MAP-2 is described as a ubiquitous cytoskeletal neuronal protein that maintains microtubule stability and is specifically found in dendritic arbours of neurons (Binder et al. 1984). Quantification was carried out in a similar manner as that described for pERK1. Differences included the use of $20 \times$ images of the CA1 SR, the CA3 SL, and the DG granule cell molecular layer for analysis, and no size restrictions on stained objects.

\section{References}

Adams JP, Roberson ED, English JD, Selcher JC, Sweatt JD. 2000. MAPK regulation of gene expression in the central nervous system. Acta Neurobiol Exp (Wars) 60: 377-394.

Ahlander M, Misane I, Schott PA, Ogren SO. 1999. A behavioral analysis of the spatial learning deficit induced by the NMDA receptor antagonist MK-801 (dizocilpine) in the rat. Neuropsychopharmacology 21: 414-426.

Amaral DG, Dent JA. 1981. Development of the mossy fibers of the dentate gyrus: I. A light and electron microscopic study of the mossy fibers and their expansions. J Comp Neurol 195: 51-86.

Ambrogi Lorenzini C, Bucherelli C, Giachetti A, Tassoni G. 1987. Spontaneous and conditioned behavior of Wistar and Long Evans rats. Arch Ital Biol 125: 155-170.

Andrews JS, Jansen JHM, Linders S, Princen A, Broekkamp CLE. 1995. Performance of four different rat strains in the autoshaping, two-object discrimination, and swim maze tests of learning and memory. Physiol Behav 57: 785-790.

Bannerman DM, Good MA, Butcher SP, Ramsay M, Morris RGM. 1995. Distinct components of spatial learning revealed by prior training and NMDA receptor blockade. Nature 378: 182-186.

Binder LI, Frankfurter A, Kim H, Caceres A, Payne MR, Rebhun LI. 1984. Heterogeneity of microtubule-associated protein 2 during rat brain development. Proc Natl Acad Sci 81: 5613-5617.

Boothe RG, Greenough WT, Lund JS, Wrege K. 1979. A quantitative investigation of spine and dendritic development of neurons in visual cortex (area 17) of Macaca nemestrina monkeys. J Comp Neurol 186: $473-489$.
Bortolotto ZA, Nistico R, More JC, Jane DE, Collingridge GL. 2005. Kainate receptors and mossy fiber LTP. Neurotoxicology 26: 769-777.

Brown J, Cooper-Kuhn CM, Kempermann G, Van Praag H, Winkler J, Gage FH, Kuhn HG. 2003. Enriched environment and physical activity stimulate hippocampal but not olfactory bulb neurogenesis. Eur J Neurosci 17: 2042-2046.

Cain DP, Saucier D, Hall J, Hargreaves EL, Boon F. 1996. Detailed behavioral analysis of water maze acquisition under APV or CNQX: Contributions of sensorimotor disturbances to drug-induced acquisition deficits. Behav Neurosci 110: 86-102.

Cain DP, Saucier D, Boon F. 1997. Testing hypotheses of spatial learning: The role of NMDA receptors and NMDA-mediated long-term potentiation. Behav Brain Res 84: 179-193.

Cassel J-C, Cassel S, Galani R, Kelche C, Will B, Jarrard LE. 1998. Fimbria-fornix vs selective hippocampal lesions in rats: Effects on locomotor activity and spatial learning and memory. Neurobiol Learn Mem 69: 22-45.

Chen Y, Bourne J, Pieribone VA, Fitzsimonds RM. 2004. The role of actin in the regulation of dendritic spine morphology and bidirectional synaptic plasticity. Neuroreport 15: 829-832.

Devan BD, White NM. 1999. Parallel information processing in the dorsal striatum: Relation to hippocampal function. J Neurosci 19: 2789-2798.

Dunaevsky A, Tashiro A, Majewska A, Mason C, Yuste R. 1999. Developmental regulation of spine motility in the mammalian central nervous system. Proc Natl Acad Sci 96: 13438-13443.

Duva CA, Floresco SB, Wunderlich GR, Lao TL, Pinel JPJ, Phillips AG. 1997. Disruption of spatial but not object-recognition memory by neurotoxic lesions of the dorsal hippocampus in rats. Behav Neurosci 111: 1184-1196.

Edwards FA. 1995. LTP-a structural model to explain the inconsistencies. Trends Neurosci 18: 250-255.

Engert F, Bonhoeffer T. 1999. Dendritic spine changes associated with hippocampal long-term synaptic plasticity. Nature 1: 1-5.

Fordyce DE, Bhat RV, Baraban JM, Wehner JM. 1994. Genetic and activity-dependent regulation of zif268 expression: Association with spatial learning. Hippocampus 4: 559-568.

Gaarskjaer FB. 1985. The development of the dentate area and the hippocampal mossy fiber projection of the rat. J Comp Neurol 241: 154-170.

Gaarskjaer FB. 1986. The organization and development of the hippocampal mossy fiber system. Brain Res 396: 335-357.

Goldin M, Segal M. 2003. Protein kinase C and ERK involvement in dendritic spine plasticity in cultured rodent hippocampal neurons. Eur J Neurosci 17: 2529-2539.

Greenough WT, Volkmar FR. 1973. Pattern of dendritic branching in occipital cortex of rats reared in complex environments. Exp Neurol 40: 491-504

Greenough WT, Volkmar FR, Juraska JM. 1973. Effects of rearing complexity on dendritic branching in frontolateral and temporal cortex of the rat. Exp Neurol 41: 371-378.

Greenough WT, Juraska JM, Volkmar FR. 1979. Maze training effects on dendritic branching in occipital cortex of adult rats. Behav Neural Biol 26: $287-297$

Hastings NB, Gould E. 1999. Rapid extension of axons into the CA3 region by adult-generated granule cells. J Comp Neurol 413: 146-154.

Hastings NB, Seth MI, Tanapat P, Rydel TA, Gould E. 2002. Granule neurons generated during development extend divergent axon collaterals to hippocampal area CA3. J Comp Neurol 452: 324-333.

Heale V, Harley C. 1990. MK-801 and AP5 impair acquisition, but not retention, of the Morris milk maze. Pharmacol Biochem Behav 36: 145-149.

Holahan MR, Taverna FA, Emrich SM, Louis M, Muller RU, Roder JC, McDonald RJ. 2005. Impairment in long-term retention but not short-term performance on a water maze reversal task following hippocampal or mediodorsal striatal $N$-methyl-D-aspartate receptor blockade. Behav Neurosci 119: 1563-1571.

Holahan MR, Rekart JL, Sandoval J, Routtenberg A. 2006. Spatial learning induces presynaptic structural remodeling in the hippocampal mossy fiber system of two rat strains. Hippocampus 16: 560-570.

Holahan MR, Honegger KS, Routtenberg A. 2007. Expansion and retraction of hippocampal mossy fibers during postweaning development: Strain-specific effects of NMDA receptor blockade. Hippocampus 17: $58-67$.

Issa AM, Rowe W, Gauthier S, Meaney MJ. 1990. Hypothalamic-pituitaryadrenal activity in aged, cognitively impaired and cognitively unimpaired rats. J Neurosci 10: 3247-3254.

Keith JR, Rudy JW. 1990. Why NMDA-receptor-dependent long-term potentiation may or may not be a mechanism of learning and memory: Reappraisal of the NMDA-receptor blockade strategy. Psychobiology 18: $251-257$

Kempermann G, Kuhn HG, Gage FH. 1997. More hippocampal neurons in adult mice living in an enriched environment. Nature 386: 493-495. 
Knierim JJ. 2006. Neural representations of location outside the hippocampus. Learn Mem 13: 405-415.

Kolb B, Robbins T. 2003. The rodent prefrontal cortex. Behav Brain Res 146: $1-2$.

Leclerc N, Baas PW, Garner CC, Kosik KS. 1996. Juvenile and mature MAP2 isoforms induce distinct patterns of process outgrowth. Mol Biol Cell 7: 443-455.

Maletic-Savatic M, Malinow R, Svoboda K. 1999. Rapid dendritic morphogenesis in CA1 hippocampal dendrites induced by synaptic activity. Science 283: 1923-1927.

Marcondes FK, Bianchi FJ, Tanno AP. 2002. Determination of the estrous cycle phases of rats: Some helpful considerations. Braz J Biol 62: 609-614.

Mazzucchelli C, Vantaggiato C, Ciamei A, Fasano S, Pakhotin P, Krezel W, Welzl H, Wolfer DP, Pages G, Valverde O, et al. 2002. Knockout of ERK1 MAP kinase enhances synaptic plasticity in the striatum and facilitates striatal-mediated learning and memory. Neuron $\mathbf{3 4}$ 807-820.

McDonald RJ, White NM. 1994. Parallel information processing in the water maze: Evidence for independent memory systems involving dorsal striatum and hippocampus. Behav Neural Biol 61: 260-270.

McDonald RJ, White NM. 1995. Hippocampal and nonhippocampal contributions to place learning in rats. Behav Neurosci 109: 579-593.

McDonald RJ, Hong NS, Devan BD. 2004. The challenges of understanding mammalian cognition and memory-based behaviours: An interactive learning and memory systems approach. Neurosci Biobehav Rev 28: $719-745$.

Meaney MJ, Aitken DH. 1985. The effects of early postnatal handling on hippocampal glucocorticoid receptor concentrations: Temporal parameters. Brain Res 354: 301-304.

Morris RGM. 1989. Synaptic plasticity and learning: Selective impairment of learning in rats and blockade of long-term potentiation in vivo by the $N$-methyl-D-aspartate receptor antagonist AP5. J Neurosci 9: 3040-3057.

Morris RGM, Garrund P, Rawlins JNP, O'Keefe J. 1982. Place navigation impaired in rats with hippocampal lesions. Nature 297: 681-683.

Moser M-B, Moser EI, Forrest E, Andersen P, Morris RGM. 1995. Spatial learning with a minislab in the dorsal hippocampus. Proc Natl Acad Sci 92: $9697-9701$.

Nilsson M, Perfilieva E, Johansson U, Orwar O, Eriksson PS. 1999. Enriched environment increases neurogenesis in the adult rat dentate gyrus and improves spatial memory. J Neurobiol 39: 569-578.

Ohno M, Yamamoto T, Watanabe S. 1992. Effects of intrahippocampal injections of $N$-methyl-D-aspartate receptor antagonists and scopolamine on working and reference memory assessed in rats by a three-panel runway task. J Pharmacol Exp Ther 263: 943-950.

Oswald CJP, Bannerman DM, Yee BK, Rawlins JNP, Honey RC, Good M. 2003. Entorhinal cortex lesions disrupt the transition between the use of intra- and extramaze cues for navigation in the water maze. Behav Neurosci 117: 588-595.

Otnaess MK, Brun VH, Moser MB, Moser EI. 1999. Pretraining prevents spatial learning impairment after saturation of hippocampal long-term potentiation. J Neurosci 19: RC49.

Paxinos G, Watson C. 1998. The rat brain atlas in stereotaxic coordinates. Academic Press, New York.

Ramirez-Amaya V, Escobar ML, Chao V, Bermudez-Rattoni F. 1999 Synaptogenesis of mossy fibers induced by spatial water maze overtraining. Hippocampus 9: 631-636.

Ramirez-Amaya V, Balderas I, Sandoval J, Escobar ML, Bermudez-Rattoni F. 2001. Spatial long-term memory is related to mossy fiber synaptogenesis. J Neurosci 21: 7340-7348.

Rauch SL, Raskin LA. 1984. Cholinergic mediation of spatial memory in the preweanling rat: Application of the radial arm maze paradigm. Behav Neurosci 98: 35-43.

Rekart JL, Sandoval CJ, Bermudez-Rattoni F, Routtenberg A. 2007a. Remodeling of hippocampal mossy fibers is selectively induced seven days after the acquisition of a spatial but not a cued reference memory task. Learn Mem 14: 416-421.

Rekart JL, Sandoval CJ, Routtenberg A. 2007b. Learning-induced axonal remodeling: Evolutionary divergence and conservation of two components of the mossy fiber system within Rodentia. Neurobiol Learn Mem 87: 225-235.
Riekkinen M, Riekkinen PJ. 1997. Dorsal hippocampal muscarinic acetylcholine and NMDA receptors disrupt water maze navigation. Neuroreport 8: 645-648.

Robinson GSJ, Crooks GBJ, Shinkman PG, Gallagher M. 1989. Behavioral effects of MK-801 mimic deficits associated with hippocampal damage. Psychobiology 17: 156-164.

Roskoden T, Linke R, Schwegler H. 2005. Transient early postnatal corticosterone treatment of rats leads to accelerated aquisition of a spatial radial maze task and morphological changes in the septohippocampal region. Behav Brain Res 157: 45-53.

Rudy JW, Stadler-Morris S, Albert P. 1987. Ontogeny of spatial navigation behaviors in the rat: Dissociation of 'proximal'- and'distal'-cue-based behaviors. Behav Neurosci 101: 62-73.

Sandi C, Davies HA, Cordero MI, Rodriguez JJ, Popov VI, Stewart MG. 2003. Rapid reversal of stress induced loss of synapses in CA3 of rat hippocampus following water maze training. Eur J Neurosci 17: 2447-2456.

Saucier D, Cain DP. 1995. Spatial learning without NMDA receptor-dependent long-term potentiation. Nature 378: 186-189.

Saucier D, Hargreaves EL, Boon F, Vanderwolf CH, Cain DP. 1996. Detailed behavioral analysis of water maze acquisition under systemic NMDA or muscarinic antagonism: Nonspatial pretraining eliminates spatial learning deficits. Behav Neurosci 110: 103-116.

Shapiro ML, Caramanos Z. 1990. NMDA antagonist MK-801 impairs acquisition but not performance of spatial working memory and reference memory. Psychobiology 18: 231-243.

Steele RG, Morris RGM. 1999. Delay-dependent impairment of a matching-to-place task with chronic and intrahippocampal infusion of the NMDA-antagonist D-AP5. Hippocampus 9: 118-136.

Sutherland RJ, Kolb B, Whishaw IQ. 1982. Spatial mapping: Definitive disruption by hippocampal or medial frontal cortical damage in the rat. Neurosci Lett 31: 271-276.

Sutherland RJ, Whishaw IQ, Kolb B. 1983. A behavioural analysis of spatial localization following electrolytic, kainate- or colchicine-induced damage to the hippocampal formation in the rat. Behav Brain Res 7: $133-153$.

Tashiro A, Makino H, Gage FH. 2007. Experience-specific functional modification of the dentate gyrus through adult neurogenesis: A critical period during an immature stage. J Neurosci 27: 3252-3259.

Uekita T, Okaichi H. 2009. Pretraining does not ameliorate spatial learning deficits induced by intrahippocampal infusion of AP5. Behav Neurosci 123: $520-526$.

Uekita T, Okaichi Y, Okaichi H. 2006. Dissociation of the roles of NMDA receptor and hippocampus in rats' spatial learning: The effects of environmental familiarity and task familiarity. Rev Neurosci 17: $163-173$.

van Praag H, Christie BR, Sejnowski TJ, Gage FH. 1999. Running enhances neurogenesis, learning, and long-term potentiation in mice. Proc Natl Acad Sci 96: $13427-13431$.

van Praag H, Kempermann G, Gage FH. 2000. Neural consequences of environmental enrichment. Nat Rev Neurosci 1: 191-198.

Volkmar FR, Greenough WT. 1972. Rearing complexity affects branching of dendrites in the visual cortex of the rat. Science 176: 1445-1447.

Warren SG, Juraska JM. 1997. Spatial and nonspatial learning across the rat estrous cycle. Behav Neurosci 111: 259-266.

Warren SG, Humphreys AG, Juraska JM, Greenough WT. 1995. LTP varies across the estrous cycle: Enhanced synaptic plasticity in proestrus rats. Brain Res 703: $26-30$.

Whishaw IQ, Jarrard LE. 1996. Evidence for extrahippocampal involvement in place learning and hippocampal invovlement in path integration. Hippocampus 6: 513-524.

Whishaw IQ, Cassel J-C, Jarrard LE. 1995. Rats with fimbria-fornix lesions display a place response in a swimming pool: A dissociation between getting there and knowing where. J Neurosci 15: 5779-5788.

Wu GY, Deisseroth K, Tsien RW. 2001. Spaced stimuli stabilize MAPK pathway activation and its effects on dendritic morphology. Nat Neurosci 4: $151-158$.

Zaharia MD, Kulczycki J, Shanks N, Meaney MJ, Anisman H. 1996. The effects of early postnatal stimulation on Morris water-maze acquisition in adult mice: Genetic and maternal factors. Psychopharmacology (Berlin) 128: $227-239$.

Received April 26, 2010; accepted in revised form May 19, 2010. 


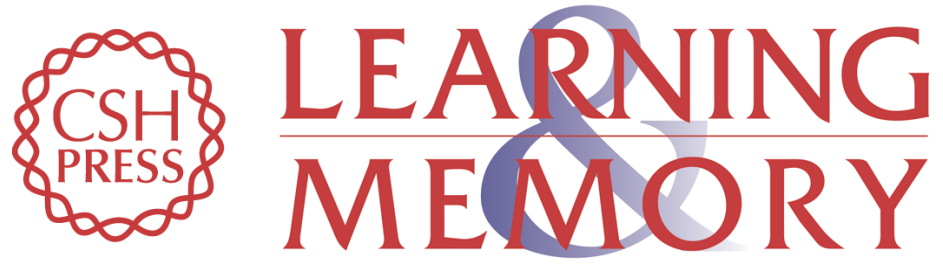

\section{Effect of juvenile pretraining on adolescent structural hippocampal attributes as a substrate for enhanced spatial performance}

Robin J. Keeley, Brianne C. Wartman, Alexander N. Häusler, et al.

Learn. Mem. 2010, 17:

Access the most recent version at doi:10.1101//m.1849910

Supplemental
Material http://learnmem.cshlp.org/content/suppl/2010/06/30/17.7.344.DC1

References This article cites 80 articles, 17 of which can be accessed free at: http://learnmem.cshlp.org/content/17/7/344.full.html\#ref-list-1

License

Email Alerting Receive free email alerts when new articles cite this article - sign up in the box at the Service top right corner of the article or click here. 